\title{
Outcomes after Cryoballoon or Radiofrequency Ablation for Persistent Atrial Fibrillation: a Multicentric Propensity-score Matched Study
}

Serge Boveda MD (1)* Rui Providência MD PhD (2), Pascal Defaye MD PhD (3), Dominque Pavin MD (4), Jean-Pierre Cebron MD ${ }^{(5)}$, Frederic Anselme MD PhD (6), Ziad Khoueiry MD (7), Nicolas Combes MD (1), Stephane Combes MD (1), Sophie Jacob (8), Jean-Paul Albenque MD (1), Pedro Sousa MD (1)

(1) Département de Rythmologie, Clinique Pasteur, Toulouse, France ; ${ }^{(2)}$ Barts Heart Centre, Barts Health NHS Trust, London, United Kingdom; (3) University Hospital of Grenoble, Grenoble, France; (4) Hospital Pontchaillou of Rennes, Rennes, France ; ${ }^{(5)}$ Nouvelles Cliniques Nantaises, Nantes, France ; ${ }^{(6)}$ University Hospital of Rouen, Rouen, France; ${ }^{(7)}$ University Hospital of Montpellier, Montpellier, France; ${ }^{(8)}$ Institut de Radioprotection et de Sureté Nucléaire, PRP-HOM, SRBE, LEPID, Laboratoire d'Epidémiologie, Paris, France.

Short Title: Cryo or RF for Persistent-AF

\section{* Corresponding author:}

Serge Boveda

Département de Rhythmologie, Clinique Pasteur

45 avenue de Lombez, BP 27617,

31076 Toulouse Cedex 3, France

Mobile phone: +33640330065

Fax: +33562211641

Email: s.boveda@clinique-pasteur.com

Original Article

Word Count: 5025 (including references and figure legends)

Disclosures and Conflicts of Interest -Boveda S receives consulting fees from Medtronic and Boston Scientific. Defaye P receives consulting fees from Medtronic, Boston Scientific and St Jude Medical. Pavin D receives compensations for teaching purposes and proctoring from Medtronic and receives consulting fees from St Jude Medical. Cebron JP receives consulting fees from St Jude Medical and Medtronic. Anselme F receives consulting fees from Medtronic and St Jude Medical, and research grant from Medtronic. Albenque JP receives consulting fees from St Jude Medical and Biosense Webster.

Acknowledgements: none

Funding: This work was supported by the Toulouse Association for the Study of Rhythm Disturbances. 


\section{Abstract}

Purpose: Recent data show no benefit of additional ablation beyond pulmonary vein isolation (PVI) in persistent atrial fibrillation (AF). Evidence suggests that radiofrequency energy (RF) and cryoballoon (CRYO) have comparable efficacy for PVI. We aimed to assess the outcomes after a single catheter ablation procedure, comparing PVI using CRYO vs. RF ablation for PVI plus additional ablation in a cohort of patients with persistent AF.

Methods: In this prospective multicenter propensity score-matched comparison, 59 consecutive patients undergoing CRYO ablation of persistent AF were matched to 59 patients treated with RF from November 2010 to June 2012

Results: During a mean follow-up of $15.6 \pm 11.5$ months, $43.2 \%$ of patients presented atrial arrhythmia relapse after a blanking period of 3 months, which was comparable between the two groups ( $40.7 \%$ in CRYO vs. $45.8 \%$ in $\mathrm{RF}$, Log rank $\mathrm{P}=0.14$; adjusted $\mathrm{HR}=1.46,95 \% \mathrm{Cl} 0.48-4.41, \mathrm{P}=0.50)$, despite the fact that $52.5 \%$ of RF patients add additional complex fractionated atrial electrogram ablation, as well as left atrial linear ablation in over twothirds (roof line in $67.8 \%$ and mitral isthmus in $32.2 \%$ ). Patients undergoing RF ablation presented a numerically, but non-significantly, lower complication rate $(5.1 \%$ vs $10.2 \%, P=0.51)$.

Conclusion: In our multicenter experience, freedom from atrial arrhythmias was comparable among matched patients treated with $\mathrm{CRYO}$ and RF, despite non-significant trends in favour of RF in terms of complications, at the cost of longer procedure times.

Keywords: Atrial fibrillation; Pulmonary vein isolation; Arrhythmia relapse; Cryoballoon ablation; Radiofrequency ablation.

Abbreviations: PVI - Pulmonary Vein Isolation; SR - Sinus Rhythm; CFAE - Complex Fractionated Atrial Electrograms; CRYO - Cryoballoon; AAD - Anti-Arrhythmic Drug; AT - Atrial Tachycardia; PNP - Phrenic Nerve Palsy. 


\section{Background}

Percutaneous catheter ablation is an established treatment option for patients with symptomatic drugrefractory atrial fibrillation (AF) [1]. Pulmonary vein isolation (PVI) is the cornerstone of AF ablation [1] and although effective in maintaining sinus rhythm (SR) in patients with paroxysmal AF, it appears to have limited success in persistent AF [1-3]. Initial data have suggested that additional line ablation [4] or complex fractionated atrial electrograms (CFAE) ablation [5] could decrease arrhythmia recurrence. However, recent results of meta-analyses [6] and randomized controlled trials [7] did not confirm the benefit of additional ablation beyond PVI in the outcome of persistent AF ablation, emphasizing the importance of durable PVI, even in persistent $A F$.

Cryoballoon (CRYO) ablation as emerged as an alternative approach to radiofrequency (RF) ablation and has proved to be at least equivalent for PVI in patients with paroxysmal AF [8-10]. The relative simplicity and faster learning curve associated with this approach have led to widespread adoption of this technology in clinical practice $[8,10]$.

Until now, only sparse data are available comparing the 1-year clinical outcome between the two techniques in patients with persistent AF. In this prospective, multicenter comparison we evaluated the outcomes after a single catheter ablation procedure, using the first generation CRYO for PVI versus open-irrigated RF ablation through a "stepwise approach" in a cohort of patients with persistent AF.

\section{Methods}

\section{Setting and Study Population}

Data concerning all consecutive patients undergoing a first procedure of cryoballoon ablation for persistent $\mathrm{AF}$ ablation in six centres from a French ablation Survey (NCT01918670-FrenchAF) were prospectively retrieved. This survey gathered tertiary public Universitary and Private centers all referent for AF ablation. Basically, FrenchAF included all consecutive patients older than 18 undergoing catheter ablation of paroxysmal and persistent AF refractory to at least one anti-arrhythmic drug agent in any of the participant centres from November 2010 to June 2012. Data regarding all patients within the specified time window was prospectively collected and inserted into each Centre's database in the day of the procedure. 
The subgroup of persistent AF patients treated with CRYO was selected for this study. In the RF-treatment arm, to be eligible, a standard RF catheter ablation procedure (point-by-point or dragging) using a 3-D mapping system had to be performed. Patients undergoing RF ablation with single-shot techniques like Ablation Frontiers, (Medtronic $\left(\right.$ ), Minneapolis, MN, USA) or NMARQ ${ }^{\mathrm{TM}}$, (Biosense Webster@ , South Diamond Bar, CA, USA), or with a previous AF ablation procedure were excluded from analysis.

The second-generation Artic Front Advance only became available in France after the inclusion period of the study (June 2012) and contact-force sensing catheters were being used at that time in only one of the centers. In order to homogenize the groups and make them comparable, we decided only to compare first generation CRYO vs. non-contact-force open-irrigated RF, and hence, patients treated with contact-force sensing RF catheters were excluded from analysis.

All participant centers had been performing RF for more than 10 years and CRYO had been in use since 2008 in two centers and 2010 in the remainder.

\section{Sample Characterization}

All variables at the time of the procedure were defined and categorized according to the literature or common practice. Persistent AF was defined as continuous AF sustained beyond 7 days or when a decision was made to cardiovert the patient after $\geq 48$ hours of $A F$, but prior to 7 days [1]. Patients with longstanding persistent $A F$ (current episode continuously lasting for more than one year) [1] were also considered eligible. AF duration was defined as the total duration since the initial AF diagnosis (either paroxysmal and/or persistent) has been made.

Valvular cardiomyopathy was defined as presence of one of the following: moderate or severe aortic or mitral valve regurgitation, any degree of aortic or mitral valve stenosis and/or previous cardiac valve replacement/repair.

Information was collected regarding demographics, anthropometric data, baseline thromboembolic and bleeding risk, anti-arrhythmic drugs (AADs) prior to the procedure, atrial dilation, left ventricular ejection fraction and presence of structural heart disease.

\section{Ablation Procedure}


Data regarding the ablation procedure was recorded. The choice of pre-procedural imaging (i.e. transoesophageal echocardiogram, multidetector cardiac computed tomography and/or cardiac magnetic resonance imaging), type of anesthesia (general anesthesia vs. conscious sedation), choice of ablation technique for every particular patient was left at the discretion of the different participating centers.

PVI was the endpoint in all procedures. Data was collected regarding procedural and fluoroscopy duration, use of 3D mapping systems, ablation technique and AADs at hospital discharge.

\section{Cryoballoon ablation}

A 14-French deflectable sheath (FlexCath ${ }^{\circledR}$ Medtronic (C) was introduced into the left atrium (LA) after a single transseptal puncture. Then the Artic Front ${ }^{\mathrm{TM}}$ (Medtronic@) balloon was introduced in the sheath, inflated, and advanced to the ostium of each pulmonary vein (PV) and ablation of PV antra was performed with at least two applications of $240 \mathrm{~s}$ per vein. Use of the $28 \mathrm{~mm}$ CRYO was recommended but left to each physician's choice. Occlusion of each vein was assessed with venous angiography. Continuous monitoring of the phrenic nerve during ablation of the right PVs was systematically performed through right phrenic nerve pacing using a quadripolar catheter placed in the superior vena cava.

In the event of failure to restore SR while ablating, direct-current cardioversion was performed in the end of the procedure. PVI was assessed using a circular catheter after two applications. If the PVs remained connected, additional applications were performed using different angulations. Durable PVI was checked 20 min after the last ablation.

\section{Radiofrequency ablation}

A single or dual transseptal approach was used at the discretion of the operator. LA geometry was collected using a circular mapping catheter guided by 3D electroanatomic mapping system (Carto 3, Biosense Webster $C$ or EnSite NavX, St. Jude MedicalC, St. Paul, MN, USA). Wide antral circumferential ablation was performed using a 4-mm irrigated-tip, non-contact force sensing catheter. PVs were isolated at the level of their antrum by creating a circular continuous lesion in a point-by-point fashion. A ten-pole circular catheter permitted to assess PV isolation. Performing additional CFAE ablation was left at the discretion of the operator. When sinus rhythm (SR) was not restored, according to physician's strategy based on the analysis of the maps provided by 
the CARTO or the NavX systems, linear lesions were deployed into the LA: roof line, mitral isthmus line, posterior line (joining the left inferior PV to the right inferior PV willing to isolate the LA posterior wall), septal line and inferior line. Pre-settings used were $30 \mathrm{~W} / 48^{\circ} \mathrm{C} / 20 \mathrm{cc} / \mathrm{min}$ except for the posterior line for which $25 \mathrm{~W}$ maximum were delivered. Whenever, AF converted into atrial tachycardia (AT); this last was mapped and ablated. When SR was restored by catheter ablation either directly from AF or through an AT, the procedure was stopped. If AT could not be converted by catheter ablation into SR, the procedure was, and SR obtained by direct-current cardioversion (DCCV). Similarly, if AF was neither converted into AT nor into SR at the end of the procedure, SR was also restored by DCCV.

RF ablation of the cavotricuspid isthmus was performed in both groups at the discretion of the operator, if typical flutter had been previously identified or if the arrhythmia organized into an isthmus dependent atrial flutter.

Once the patient was in SR, bidirectional block was systematically assessed at the PVs antra, the cavo-tricuspid isthmus, the LA roof and the mitral isthmus.

\section{Follow-up and Outcomes}

Patients could be discharged on anti-arrhythmic agents, according to investigator's preference, but these were stopped after the first 3 months. The first three months post-procedure were classified as a blanking period [11]. The primary endpoint was AF/atrial tachycardia (AT) recurrence, defined as any symptomatic or asymptomatic atrial arrhythmia lasting >30 seconds after the blanking period. However, if a patient relapsed during the blanking period and remained in AF/AT despite all attempts to restore SR, this was also considered a procedural failure. AF/AT relapse before discharge, and relapse during the 3-months blanking period were also systematically assessed.

The following monitoring protocol was proposed after discharge: a clinical assessment either at the ablating center or with patient's local cardiologist including a 12-lead ECG and a 24-hour Holter at 1, 3, 6, 9, and 12 months after the procedure. Following that, one consultation per year with an ECG and a 24-hour Holter was also suggested. In the event of a patient developing symptoms suggestive of relapse, the recommendation was to perform a 12-lead ECG as soon as possible and if this failed to document the arrhythmia, a 24-hour Holter or 
an external loop recorder were advised. In patients with previously implanted intracardiac rhythm management devices these were used for monitoring AF/AT relapses.

With regard to safety, the following complications were systematically screened: vascular complications (if requiring intervention or prolongation of admission), thromboembolism (transient ischemic attack, stroke and/or systemic embolism), phrenic nerve palsy (PNP) persisting after the procedure, pericardial effusion (if causing haemodynamic instability and/or requiring pericardiocenthesis or prolonged monitoring), and procedure-related death.

ECG and Holter tracings were organized and checked by the locally and adjudication of relapse or procedural complications was done by the local electrophysiologist investigator.

\section{$\underline{\text { Statistical analysis }}$}

Chi-square was used for the comparison of nominal variables. The t-student test and one-way ANOVA, or their non-parametric equivalents, Mann-Whitney and Kruskall-Wallis when appropriate, were used for comparison of continuous variables; the Levene's test was used in order to check the homogeneity of variance. Results with $\mathrm{P}<0.05$ were regarded as significant.

A propensity score was obtained for all participants undergoing a first procedure of persistent AF ablation through binary logistic regression: ablation technique (RF or CRYO) was the binary outcome and all baseline variables were used as covariates for estimating a probability (the propensity score). Then, probabilities in the CRYO group were matched 1:1 to the best RF corresponding patient using the nearest neighbour matching approach. Histograms and box-plots, and comparison of means and medians, were used for assessing distribution and matching success.

Comparisons between RF and CRYO were then performed. Kaplan-Meier curves were traced for comparing sinus rhythm maintenance among the two intervention groups. The log rank test was used for assessing the existence of differences. The propensity-matched hazard ratio for this comparison was estimated after adjustment for all baseline variables whose comparison had shown a $\mathrm{P}$ of $<0.5$ (Multivariate Cox Regression, Method: Enter).

In propensity matched pairs, Univariate Cox and multivariate Cox regressions (Method: forward likelihood ratio, probability for stepwise $=0.05$ ) were performed for assessing for possible predictors of atrial arrhythmia relapse after blanking. 
Data was filled into a pre-defined data introduction electronic sheet made available to all participant Centers. After completion of follow-up, data from all Centers was merged and analysed at the Coordinating Center (Clinique Pasteur, Toulouse).

PASW Statistics (SPSS Inc, Chicago, IL) version 18.0 was used for descriptive and inferential statistical analysis.

G*Power 3.1.2. was used for power assessment of the sample.

\section{Results}

\section{Study population}

Among 622 consecutive procedures of persistent AF ablation in the FrenchAF survey, 142 were excluded as they were redo ablation procedures, and 51 procedures were also excluded as a contact-force sensing catheter was used. Among the remaining 429 patients, 59 consecutive patients undergoing CRYO ablation for persistent AF and 59 propensity score-matched controls treated with RF were included in this analysis. Mean age of the sample was $59.9 \pm 10.7$ and $17.8 \%(n=21)$ were women. The mean AF duration since diagnosis, $\mathrm{CHA}_{2} \mathrm{DS}_{2}-\mathrm{VASC}$ and HAS-BLED scores were $4.0 \pm 4.0$ years, $1.2 \pm 1.1$ and $0.6 \pm 0.7$, respectively. Figures 1 and 2 illustrate the similar distribution (absolute values and Quartiles, respectively) of the propensity score among the two treatment groups. Propensity-score matching was accurate, and no significant baseline differences were present between the two treatment arms (Table 1).

Data on patients undergoing a first procedure of persistent AF ablation is illustrated in S-Table 1 (Supplementary material). This shows that our cohort of patients treated with CRYO and, correspondingly, their RF controls, are mainly composed of a less advanced persistent AF phenotype ("early persistent AF"), has they have a lower $\mathrm{CHA}_{2} \mathrm{DS}_{2}$-VASc score, and consequently lower prevalence of congestive heart failure, hypertension and Diabetes mellitus, present in sinus rhythm more often at the start of the procedure, have less dilated left atria, and more preserved LVEF. Also, only a very small minority of our eligible cohort was composed of longstanding persistent $\mathrm{AF}$, unlike in the overall FrenchAF sample, where this was almost $15 \%$.

\section{$\underline{\text { Procedural Data }}$}

The prevalence of $A F$ at the beginning of the procedure was comparable: $78.0 \%$ RF vs. $72.9 \%$ CRYO (P=0.52). Patients undergoing CRYO ablation presented shorter procedure $(120 \pm 33$ vs. $152 \pm 61, P<0.01)$ and similar 
fluoroscopy duration ( $28 \pm 16$ vs. $36 \pm 13, P=0.27$ ) compared to the RF group (see Table 2). The large, 28mm, Artic Front ${ }^{\mathrm{TM}}$ balloon was used in all patients.

All PVs were isolated in 58 (98.3\%) patients in each group. Five patients (8.5\%) in the CRYO group required focal "touch-up" RF ablation to achieve PVI. In the RF group, $52.5 \%$ of patients $(n=31)$ had CFAE, and ablation directed to the roof and the mitral isthmus in $67.8 \%(n=40)$ and $32.2 \%(n=19)$ patients, respectively.

The cavotricuspid isthmus line was ablated in 12 patients in the RF group and in 11 patients in the CRYO group (20.3\% vs. $18.6 \%, P=0.82)$.

No differences in AADs prescription at discharge were observed between the two groups (47.5\% in RF vs. 49.2\% in CRYO, $\mathrm{P}=0.85)$.

\section{Procedural Complications}

Ten of the 118 patients (8.5\%) experienced a complication, as detailed in Table 3. Patients undergoing RF ablation presented a numerically, but non-significantly, lower complication rate $(6.8 \%$ vs $10.2 \%, P=0.51)$. PNP was only observed in 2 patients - both belonging to the CRYO group, but resolved completely before discharge.

\section{Efficacy}

No significant differences were observed among the two treatment strategies regarding relapses during the blanking period (35.6\% in RF vs. $22.0 \%$ in CRYO, $P=0.10$ ). Based on an Alpha of 0.05 , this sample of 118 individuals had a power of 0.80 (Beta 0.20 ) for showing a $40 \%$ reduction in relapse (effect size of 0.28 assuming a $50 \%$ relapse rate at 12 months), expecting $10 \%$ of individuals to be lost to follow-up during the study.

All patients had at least 6 months of follow-up, and between 6 and 12 months, 8 patients (7\%) were lost to follow-up. During mean follow-up of $15.6 \pm 11.5$ months, $43.2 \%(n=51)$ of patients presented AF/AT relapse.

Kaplan-Meier curves for freedom from AF/AT in the two treatment groups were comparable (log rank $\mathrm{P}=0.14$; adjusted $\mathrm{HR}=1.46,95 \% \mathrm{Cl} 0.48-4.41, \mathrm{P}=0.50)$, as seen in Figure 3, with $40.7 \%(\mathrm{n}=24)$ vs. $45.8 \%(\mathrm{n}=20)$ relapse rates in the CRYO and RF groups, respectively.

\section{Predictors of arrhythmia relapse}

On multivariate Cox regression (Table 4), only AF duration in years ( $\mathrm{HR}=1.10,95 \% \mathrm{Cl} 1.01-1.10, \mathrm{P}=0.04)$ was a predictor of relapse. 


\section{Discussion}

In this first propensity-score matched comparison of CRYO vs. RF for persistent AF ablation several findings are noteworthy. Until now, outcomes after CRYO ablation procedures in persistent AF patients have been evaluated indirectly by meta-analyses [9], non-randomized comparisons [10, 12], or observational studies [1315]. In this study, we have tried for the first time to compare both techniques more directly, thanks to a propensity-score matching (1:1). Indeed, even if it will not replace a randomized trial, we believe that this approach can provide a more comprehensive and objective insight of this timely question. Second, patients treated with cryoablation had shorter procedural duration in this particular indication. Third, despite the additional and more extensive ablation beyond PVI in the RF group a comparable complication rate was observed in the two treatment arms.

In this day and age, the pathophysiology and best approach for persistent AF ablation are still unclear, and therefore the cornerstone of this therapy is still the achievement of a durable PVI [7]. For that purpose, cryoablation was shown to be a viable alternative to RF.

In our data, performing additional ablation was associated with longer procedure duration. This has been found in previous meta-analyses comparing CRYO ablation with $\mathrm{RF}[9,16]$ and PVI with PVI plus additional atrial ablation [7], although this finding varies among reports published in literature.

The non-homogenous RF ablation approach (more than two thirds of patients in the RF arm received additional lesions) casts doubt on whether the energy source or the ablation strategy was responsible for the observed results in outcome. The reason why additional ablation was not associated with an increased benefit is still unclear. It could be that a substrate approach with more extensive ablation may be iatrogenic and may predispose to new areas of arrhythmogenesis due to incompletely ablated tissue or incomplete lines of conduction block $[17,18]$. On the other hand, we can hypothesize that the culprit area was not targeted and neither CFAE nor linear lesions are the ideal complementary targets for ablation [19-21] and we still need to identify accurately the "eye of the storm"- like targeting rotors [22], ganglia [23] or using other mapping algorithms $[24,25]$.

Our results for persistent $\mathrm{AF}$ ablation, both with $\mathrm{CRYO}$ and $\mathrm{RF}$, showing $56.8 \%$ freedom from atrial arrhythmias during follow-up after a single ablation were comparable to the available data. In other trials, persistent AF patients treated with CRYO ablation presented a 42-67\% freedom from atrial arrhythmias at approximately 12 
months $[8,12,26]$. Patients with persistent AF undergoing RF ablation also presented a comparable rate of freedom from AF after a single RF procedure $[7,12]$.

One consideration should also be made regarding the potential need for a RF catheter in cryoablation. Several patients have right atrial flutter associated to AF episodes. In our study, a RF catheter was used in 11 patients (18.6\%) in the CRYO group (for completing PVI or ablating the cavotricuspid isthmus). Therefore a significant percentage of patients undergoing cryoablation for persistent AF needed a second ablation catheter, resulting in increased procedural costs.

With regard to safety, the incidence of complications was comparable and PNP occurred only in the cryoablation group, and in all cases PNP was temporary. This confirms the experience of other groups performing RF and cryoablation [10, 27, 28].

Our data reinforce the role of AF duration, and consequently electrical AF remodeling ("AF begets $A F$ "), as only AF duration (years since diagnosis and longstanding persistent AF) was an independent predictor of arrhythmia relapse like previously shown by Tilz et al. [29]. Need for cardioversion at the end of the procedure was not a predictor of relapse in our sample. Studies in persistent AF patients revealed that AF termination during procedure is associated with a better outcome and failure to terminate AF was a predictor of recurrence [3032]. Those studies provided support for additional atrial ablation, as it frequently increases the rate of patients converting to sinus rhythm during the procedure.

\section{Study limitations}

We acknowledge several limitations in our investigation. First, the results of this multicenter study should be interpreted carefully in view of its non-randomized design. Nevertheless, the use of propensity-score matching provided an appropriately matched control group, minimizing that issue. Second, since this was a multicenter registry, differences in ablation strategy and peri-procedural management may have existed among centers and operators. Third, as illustrated in Table S-1, the true groups of persistent AF patients compared in this analyses represent a lower risk AF cohort, and should therefore be regarded with caution, as they may not be extrapolated to the global persistent AF population. Lastly, in this sample, only first generation Artic Front balloon and non-contact-force sensing catheters were used, as this allows a fairer comparison, as previously shown by Mugnai et al. [28], and the new generation technologies were not available at the time to all (contact-force sensing catheters) or any ( $2^{\text {nd }}$ generation cryoballoon) centers of the FrenchAF survey. In current 
practice these new generation catheters are expected to improve the freedom from atrial arrhythmia recurrence, with the $2^{\text {nd }}$ generation cryoballoon and contact-force sensing catheters presenting better results than their previous generation counterparts (non contact-force sensing catheters vs. contact-force sensing catheters [33] and $2^{\text {nd }}$ generation vs. $1^{\text {st }}$ generation cryoballoon [34, 35]), but presenting similar results in direct comparisons $[12,36,37]$. We believe that these positive evolutions don't detract the global message of our paper which emphasizes the major importance of PVI for treating persistent AF patients, but further studies are of course needed with the latest generation catheters in order to confirm our encouraging results.

\section{Conclusions}

This prospective, multicenter, propensity-score matched analysis comparing CRYO with open-irrigated RF ablation in the setting of persistent AF ablation shows that freedom from atrial arrhythmias was comparable in both treatment arms, despite non-significant trends in favour of RF in terms of complications, at the cost of longer procedure times. 


\section{References}

1. Calkins, H., Kuck, K.H., Cappato, R., Brugada, J., Camm, A.J., Chen, S.A., et al. (2012). 2012 HRS/EHRA/ECAS expert consensus statement on catheter and surgical ablation of atrial fibrillation: recommendations for patient selection, procedural techniques, patient management and follow-up, definitions, endpoints, and research trial design: a report of the Heart Rhythm Society (HRS) Task Force on Catheter and Surgical Ablation of Atrial Fibrillation. Developed in partnership with the European Heart Rhythm Association (EHRA), a registered branch of the European Society of Cardiology (ESC) and the European Cardiac Arrhythmia Society (ECAS); and in collaboration with the American College of Cardiology (ACC), American Heart Association (AHA), the Asia Pacific Heart Rhythm Society (APHRS), and the Society of Thoracic Surgeons (STS). Endorsed by the governing bodies of the American College of Cardiology Foundation, the American Heart Association, the European Cardiac Arrhythmia Society, the European Heart Rhythm Association, the Society of Thoracic Surgeons, the Asia Pacific Heart Rhythm Society, and the Heart Rhythm Society. Heart Rhythm, 9(4), 632-696.e21.

2. Tilz, R.R., Rillig, A., Thum, A.M., Arya, A., Wohlmuth, P., Metzner, A., et al. (2012). Catheter ablation of longstanding persistent atrial fibrillation: 5-year outcomes of the Hamburg Sequential Ablation Strategy. J Am Coll Cardiol, 60(19), 1921-1929.

3. Elayi, C.S., Verma, A., Di Biase, L., Ching, C.K., Patel, D., Barrett, C., et al. (2008). Ablation for longstanding permanent atrial fibrillation: results from a randomized study comparing three different strategies. Heart Rhythm, 5(12), 1658-1664.

4. Wynn, G.J., Das, M., Bonnett, L.J., Panikker, S., Wong, T., Gupta, D. (2014). A Efficacy of Catheter Ablation for Persistent Atrial Fibrillation: A Systematic Review and Meta-Analysis of Evidence From Randomized and Nonrandomized Controlled Trials. Circ Arrhythm Electrophysiol, 7(5), 841-852.

5. Verma, A., Mantovan, R., Macle, L., De Martino, G., Chen, J., Moreillo, C.A., et al. (2010). A Substrate and Trigger Ablation for Reduction of Atrial Fibrillation (STAR AF): a randomized, multicentre, international trial. Eur Heart J, 31(11), 1344-1356.

6. Providência, R., Lambiase, P.D., Srinivasan, N., Ganesha Babu, G., Bronis, K., Ahsan, S., et al. (2015). Is There Still a Role for CFAE Ablation in Addition to Pulmonary Vein Isolation in Patients with Paroxysmal and Persistent Atrial Fibrillation? A Meta-Analysis of 1,415 Patients. Circ Arrhythm Electrophysiol, 8(5), 1017-29.

7. Verma, A., Jiang, C.Y., Betts, T.R., Chen, J., Deisenhofer, I., Mantovan, R., et al. (2015). Approaches to Catheter Ablation for Persistent Atrial Fibrillation. N Engl J Med, 372(19), 1812-1822. 
8. Andrade, J.G., Khairy, P., Guerra, P.G., Deyell, M.W., Rivard, L., Macle, L., et al. (2011). Efficacy and safety of cryoballoon ablation for atrial fibrillation: A systematic review of published studies. Heart Rhythm, 8(9), 14441451.

9. Cheng, X., Hu, Q., Zhou, C., Liu, L.Q., Chen, T., Liu, Z., et al. (2015). The long-term efficacy of cryoballoon vs irrigated radiofrequency ablation for the treatment of atrial fibrillation: A meta-analysis. Int J Cardiol, 181, 297302.

10. Aryana, A., Singh, S.M., Kowalski, M., Pujara, D.K., Cohen, A.I., Singh, S.K., et al. (2015). Acute and LongTerm Outcomes of Catheter Ablation of Atrial Fibrillation Using the Second-Generation Cryoballoon versus Open-Irrigated Radiofrequency: A Multicenter Experience. J Cardiovasc Electrophysiol. 26(8), 832-9.

11. Joshi, S., Choi, A.D., Kamath, G.S., Raiszadeh, F., Marrero, D., Badheka, A., et al. (2009). Prevalence, predictors, and prognosis of atrial fibrillation early after pulmonary vein isolation: findings from 3 months of continuous automatic ECG loop recordings. J Cardiovasc Electrophysiol, 20(10), 1089-1094.

12. Ciconte, G., Baltogiannis, G., de Asmundis, C., Sieira, J., Conte, G., Di Giovanni, G., et al. (2015). Circumferential pulmonary vein isolation as index procedure for persistent atrial fibrillation: a comparison between radiofrequency catheter ablation and second-generation cryoballoon ablation. Europace, 17(4), 559565.

13. Koektuerk, B., Yorgun, H., Hengeoz, O., Turan, C.H., Dahmen, A., Yang, A., et al. (2015). Cryoballoon Ablation for Pulmonary Vein Isolation in Patients with Persistent Atrial Fibrillation: One-Year Outcome Using Second Generation Cryoballoon. Circ Arrhythm Electrophysiol 8(5), 1073-9.

14. Ciconte, G., Ottaviano, L., de Asmundis, C., Baltogiannis, G., Conte, G., Sieira, J., et al. (2015). Pulmonary vein isolation as index procedure for persistent atrial fibrillation: One-year clinical outcome after ablation using the second-generation cryoballoon. Heart Rhythm, 12(1), 60-66.

15. Lemes, C., Wissner, E., Lin, T., Mathew, S., Deiss, S., Rillig, A., et al. (2016). One-year clinical outcome after pulmonary vein isolation in persistent atrial fibrillation using the second-generation $28 \mathrm{~mm}$ cryoballoon: a retrospective analysis. Europace, 8(2):201-5.

16. Xu, J., Huang, Y., Cai, H., Qi, Y., Jia, N., Shen, W., et al. (2014). Is cryoballoon ablation preferable to radiofrequency ablation for treatment of atrial fibrillation by pulmonary vein isolation? A meta-analysis. PLos One, 28:e90323. 
17. Sawhney, N., Anousheh, R., Chen, W., Feld, G.K. (2010). Circumferential pulmonary vein ablation with additional linear ablation results in an increased incidence of left atrial flutter compared with segmental pulmonary vein isolation as an initial approach to ablation of paroxysmal atrial fibrillation. Circ Arrhythm Electrophysiol, 3(3), 242-248.

18. Shah, A.J., Jadidi, A., Liu, X., Miyazaki, S., Forclaz, A., Nault, I., et al. (2010). Atrial tachycardias arising from ablation of atrial fibrillation: a proarrhythmic bump or an antiarrhythmic turn? Cardiol Res Pract, 2010, 950763. 19. Dixit, S., Marchlinski, F.E., Lin, D., Callans, D.J., Bala, R., Riley, M.P., et al. (2012). Randomized ablation strategies for the treatment of persistent atrial fibrillation: RASTA study. Circ Arrhtyhm Electrophysiol, 5(2), 287-294

20. Shah, A.J., Pascale, P., Miyazaki, S., Liu, X., Roten, L., Derval, N., et al. (2012). Prevalence and types of pitfall in the assessment of mitral isthmus linear conduction block. Circ Arrhythm Electrophysiol, 5(5), 957-967.

21. Oral, H., Chugh, A., Yoshida, K., Sarrazin, J.F., Kuhne, M., Crawford, T., et al. (2009). A randomized assessment of the incremental role of complex fractionated atrial electrograms after antral pulmonary vein isolation for long-lasting persistent atrial fibrillation. J Am Coll Cardiol, 53(9), 782-789.

22. Pokushalov, E., Romanov, A., Artyomenko, S., Turov, A., Shugayev, P., Shirokova, N., et al. (2010). Ganglionated plexi ablation for longstanding persistent atrial fibrillation. Europace, 12(3), 342-346.

23. Narayan, S.M., Krummen, D.E., Shivkumar, K., Clopton, P., Rappel, W.J., et al. (2012). Treatment of atrial fibrillation by the ablation of localized sources: CONFIRM (Conventional Ablation for Atrial Fibrillation With or Without Focal Impulse and Rotor Modulation) trial. J Am Coll Cardiol, 60(7), 628-636.

24. Lin, Y.J., Tsao, H.M., Chang, S.L., Lo, L.W., Hu, Y.F., Chang, C.J., et al. (2010). Role of high dominant frequency sites in nonparoxysmal atrial fibrillation patients: insights from high-density frequency and fractionation mapping. Heart Rhythm, 7(9), 1255-1262.

25. Lin, Y.J., Suenari, K., Lo, M.T., Lin, C., Hsieh, W.H., Chang, S.L., et al. (2012). Novel assessment of temporal variation in fractionated electrograms using histogram analysis of local fractionation interval in patients with persistent atrial fibrillation. Circ Arrhythm Electrophysiol, 5(5), 949-956.

26. Neumann, T., Vogt, J., Schumacher, B., Dorszewski, A., Kuniss, M., Neuser, H., et al. (2008). Circumferential pulmonary vein isolation with the cryoballoon technique results from a prospective 3-center study. J Am Coll Cardiol, 52(4), 273-278. 
27. Schmidt, M., Dorwarth, U., Andresen, D., Brachmann, J., Kuck, K.H., Kuniss, M., et al. (2014). Cryoballoon versus RF ablation in paroxysmal atrial fibrillation: Results from the German Ablation Registry. J Cardiovasc Electrophysiol, 25(1), 1-7.

28. Mugnai, G., Chierchia, G.B., de Asmundis, C., Sieira-Moret, J., Conte, G., Capulzini, L., et al. (2014). Comparison of Pulmonary Vein Isolation Using Cryoballoon Versus Conventional Radiofrequency for Paroxysmal Atrial Fibrillation. Am J Cardiol, 113(9), 1509-1513.

29. Tilz, R.R., Rillig, A., Thum, A.M., Arya, A., Wohlmuth, P., Metzner, A., et al. (2012) Catheter ablation of longstanding persistent atrial fibrillation: 5-year outcomes of the Hamburg Sequential Ablation Strategy. J Am Coll Cardiol, 60(19), 1921-9.

30. O’Neill, M.D., Wright, M., Knecht, S., Jaïs, P., Hocini, M., Takahashi, Y., et al. (2009). Long-term follow-up of persistent atrial fibrillation ablation using termination as a procedural endpoint. Eur Heart J, 30(9), 1105-1112.

31. Rostock, T., Salukhe, T.V., Steven, D., Drewitz, I., Hoffmann, B.A., Bock, K., et al. (2011). Long-term singleand multiple-procedure outcome and predictors of success after catheter ablation for persistent atrial fibrillation. Heart Rhythm, 8(9), 1391-1397.

32. Schreiber, D., Rostock, T., Frolich, M., Sultan, A., Servatius, H., Hoffmann, B.A., et al. (2015). Five-year Follow-up after catheter ablation of persistent atrial fibrillation using the "stepwise approach" and prognostic factors for success. Circ Arrhythm Electrophysiol, 8(2), 308-317.

33. Marijon ,E., Fazaa, S., Narayanan, K., Guy-Moyat, B., Bouzeman, A., Providencia, R., et al. (2014). Real-time contact force sensing for pulmonary vein isolation in the setting of paroxysmal atrial fibrillation: procedural and 1-year results. J Cardiovasc Electrophysiol, 25(2), 130-7.

34.Fürnkranz, A., Bordignon, S., Dugo, D., Perotta, L., Gunawardene, M., Schulte-Hahn, B., et al. (2014) Improved 1-year clinical success rate of pulmonary vein isolation with the second-generation cryoballoon in patients with paroxysmal atrial fibrillation. J Cardiovasc Electrophysiol, 25(8), 840-4.

35. Straube, F., Dorwarth, U., Schmidt, M., Wankerl, M., Ebersberger, U., Hoffmann, E. (2014) Comparison of the first and second cryoballoon: high-volume single-center safety and efficacy analysis. Circ Arrhythm Electrophysiol, 7(2), 293-9.

36. Squara, F., Zhao, A., Marijon, E., Latcu, D.G., Providencia, R., Di Giovanni, G., et al. (2015). Comparison between radiofrequency with contact force-sensing and second-generation cryoballoon for paroxysmal atrial fibrillation catheter ablation: a multicenter European evaluation. Europace, 17(5), 718-724. 
37. Jourda, F., Providencia, R., Marijon, E., Bouzeman, A., Hireche, H., Khoueiry, Z., et al. (2015). Contact-force guided radiofrequency vs. second-generation balloon cryotherapy for pulmonary vein isolation in patients with paroxysmal atrial fibrillation - a prospective evaluation. Europace, 17(2), 225-231. 
Figure 1 - Histogram illustrating the distribution of propensity-score among the two treatment groups.

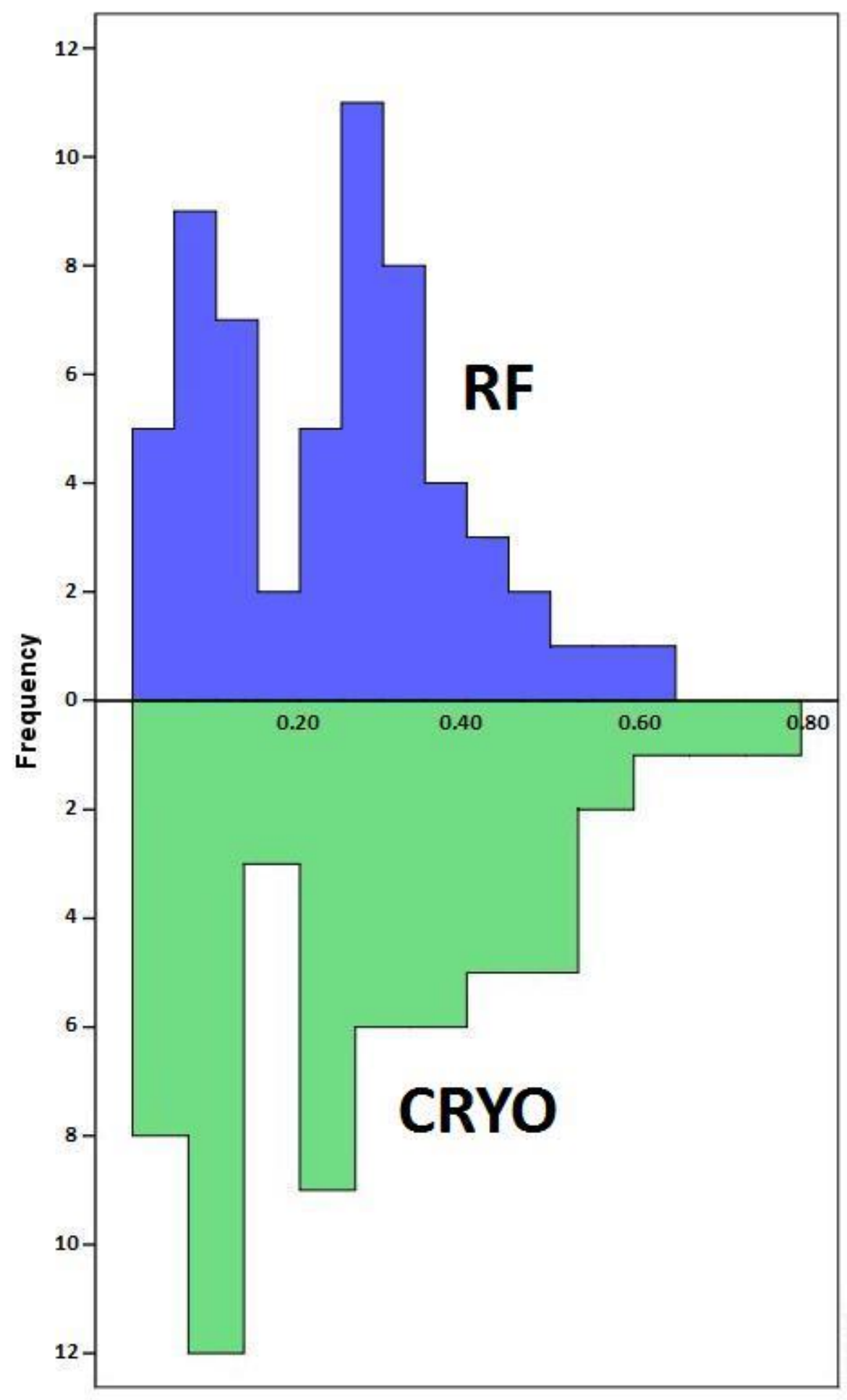

Propensity Score

Legend: Comparison of means (t-student) shows no significant differences between the two groups - RF $0.24 \pm 0.15$ vs. CRYO $0.27 \pm 0.19, P=0.28$. $R F$ - radiofrequency ablation; CRYO - cryoballoon ablation. 
Figure 2 - Box-plot illustrating the distribution of propensity-score among the two treatment groups.

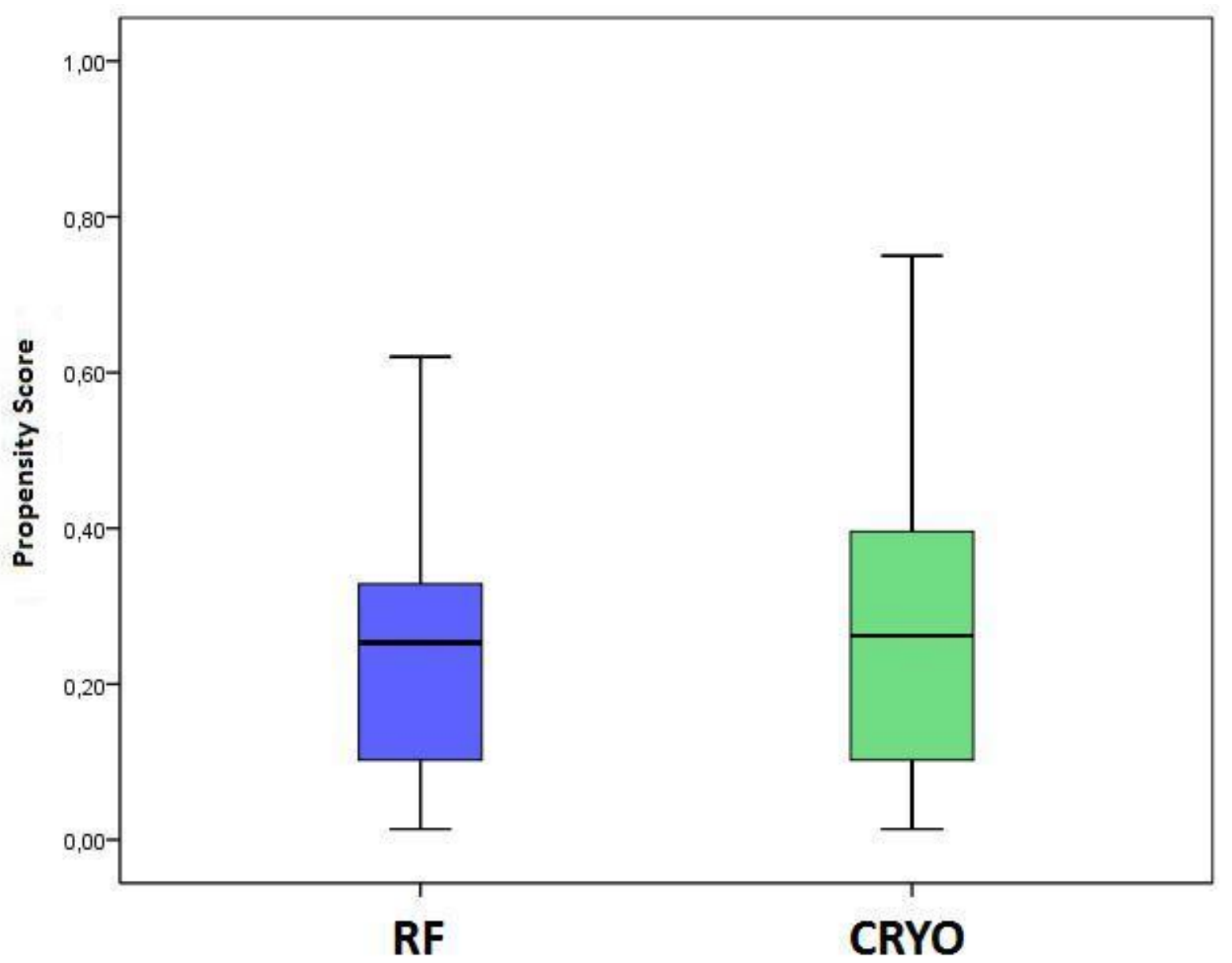

Legend: Comparison of medians (Mann-Whitney) shows no significant differences between the two treatment groups - RF $0.25(0.10-0.33)$ vs. CRYO $0.26(0.10-0.40), P=0.46$. RF - radiofrequency ablation; CRYO cryoballoon ablation. 
Figure 3 - Kaplan-Meir curve illustrating freedom from arrhythmia relapse.

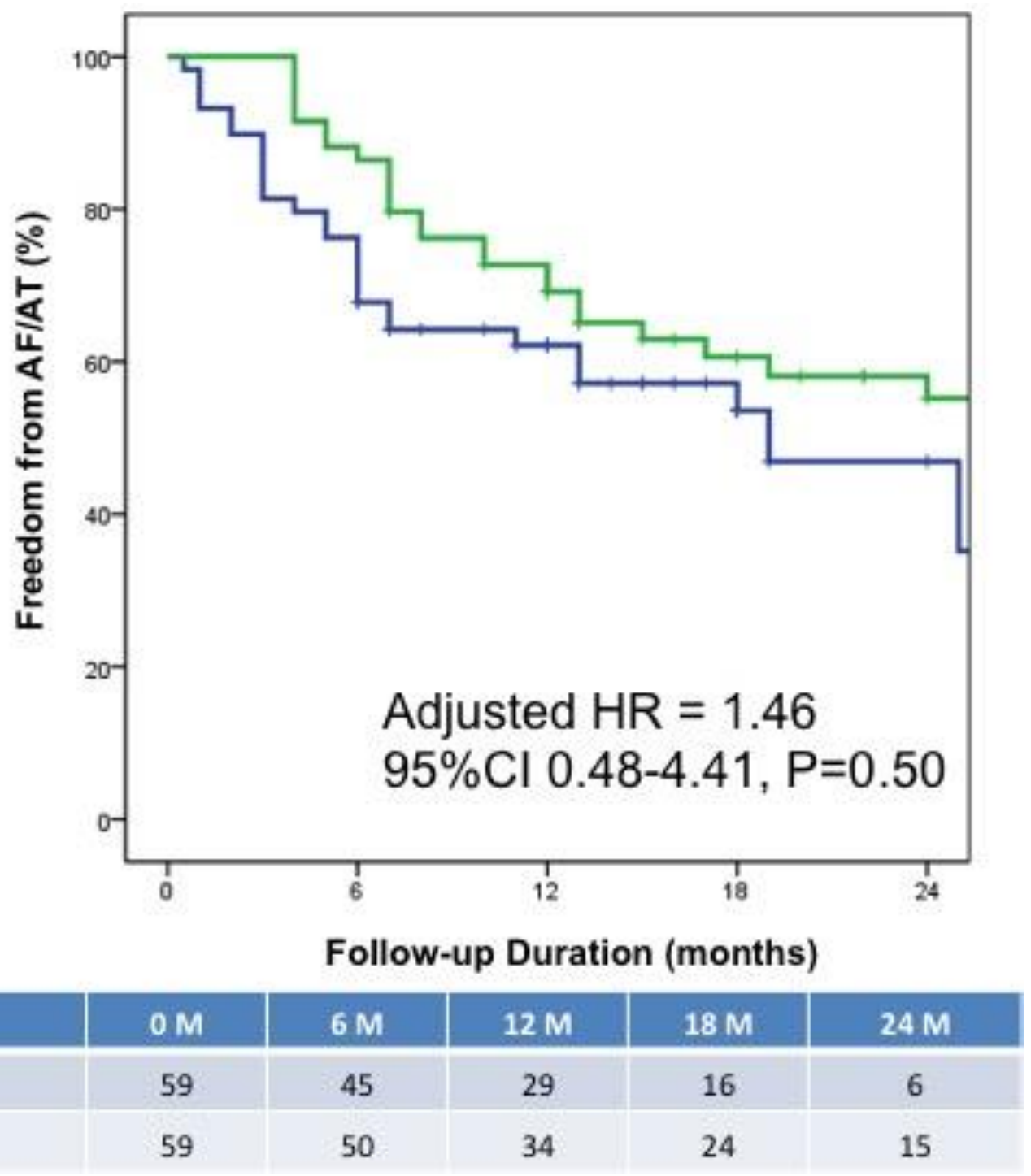

Legend: AF - atrial fibrillation; AT - atrial tachycardia; HR - hazard ratio; $\mathrm{Cl}$ - confidence interval; RF radiofrequency ablation; CRYO - cryoballoon ablation. 